\title{
Spectrum of trace deformed Yang-Mills theories
}

\author{
Andreas Athenodorou $\odot,{ }^{*}$ Marco Cardinali $\odot,{ }^{\dagger}$ and Massimo D’Elia \\ Dipartimento di Fisica, Università di Pisa and INFN, Sezione di Pisa, \\ Largo Pontecorvo 3, 56127 Pisa, Italy
}

(Received 26 December 2020; accepted 8 September 2021; published 11 October 2021)

\begin{abstract}
In this paper we study, by means of numerical simulations, the behavior of the scalar glueball mass and the ground state of the torelon for trace deformed Yang-Mills theory defined on $\mathbb{R}^{3} \times S^{1}$, in which center symmetry is recovered even at small compactification radii. We find, by investigating both $S U(3)$ and $S U(4)$ pure gauge theories, that the glueball mass computed in the deformed theory, when center symmetry is recovered, is compatible with its value at zero temperature and does not show any significant dependence on the compactification radius; moreover, we establish a connection between the deformation parameter and an effective compactification size, which works well at least for small deformations. In addition, we observe that the ground state of the torelon which winds around the small traced deformed circle with size $l$ acquires a plateau for large values of the strength $h$, with values which are compatible with a $1 / l$ behavior but, on the other hand, are still not in complete agreement with the asymptotic semiclassical large- $N$ predictions.
\end{abstract}

DOI: 10.1103/PhysRevD.104.074510

\section{INTRODUCTION}

Large- $N$ volume independence of $S U(N)$ Yang-Mills and similar theories is a topic discussed since long [1-6]. The possibility that the nonperturbative properties of the theory might be encoded in a simplified model with small compactification radii is very appealing, also in view of the fact that the inverse compactification radius sets a high energy scale, which makes weak coupling approaches viable. It has long since been clarified that volume independence holds only when no transition, leading to the breaking of center symmetry, takes place: unfortunately, the breaking happens in most cases, e.g., at the thermal radius where the theory deconfines.

More recently, trace deformed theories [7,8] have been introduced, as a way to maintain center symmetry unbroken even in the presence of arbitrarily small compactification radii. That provides a tool to study the issue of volume independence in a controlled way, e.g., by numerical lattice simulations, and also to compare with the expectations from semiclassical analytic computations. The idea, inspired by the perturbative form of the Polyakov loop effective action at high $T$ [9], is to introduce center

\footnotetext{
*andreas.athinodorou@pi.infn.it † marco.cardinali@pi.infn.it

¥massimo.delia@unipi.it
}

Published by the American Physical Society under the terms of the Creative Commons Attribution 4.0 International license. Further distribution of this work must maintain attribution to the author(s) and the published article's title, journal citation, and DOI. Funded by SCOAP . symmetric couplings to the Polyakov loop and its powers, so as to inhibit the spontaneous breaking of center symmetry. This offers the possibility to test volume independence and, at the same time, to investigate the connection of center symmetry to many other nonperturbative features of Yang-Mills theories.

Several studies have already considered the use of trace deformed theories and also of possible alternatives, like the introduction of adjoint fermions [5,6,10-25]. From the point of view of lattice simulations, recent studies have provided extensive numerical evidence regarding $\theta$-dependence in $S U(3)$ [26] and $S U(4)$ [27] gauge theories, considering in particular the first two coefficients of the Taylor expansion of the free energy expansion as a function of $\theta$. The remarkable result is that, even for compactification radii as small as $(500 \mathrm{MeV})^{-1}$, one recovers, within numerical errors, the same $\theta$-dependence as in the confined phase as soon as center symmetry is completely restored by means of the trace deformations. This goes even beyond the expectations from analytic semiclassical computations, since the volume independence, at least for what concerns $\theta$-dependence, is quantitatively exact (within errors) and observed for not-too-large values of $N$. The result is even more interesting when considering that, from a dynamical point of view, the restoration of center symmetry takes place in a slightly different way in the standard confined phase and in the deformed theory [26,27], so it seems that it is just the realization of the symmetry that counts, at least for $\theta$-dependence, independently of other details.

That claims for a deeper investigation, regarding also other nonperturbative properties of Yang-Mills theories. In 
this study we consider the physical spectrum of the theory, which for a pure gauge theory consists mostly of glueballs. The glueball spectrum is known to undergo strong modifications when crossing the deconfinement transition $[28,29]$, therefore checking if it goes back to the $T=0$ spectrum by just recovering center symmetry represents a strong test of volume independence: anticipating somewhat our conclusions and the final discussion, the answer is positive, at least for the $S U(3)$ and $S U(4)$ gauge theories which are investigated in the present study. A different issue regards the torelon masses, i.e., the masses of physical states corresponding to flux tubes winding around the compactified direction: this has no correspondence with the infinite volume theory, however there are well defined semiclassical predictions [7] that one would like to compare with.

The paper is organized as follows. In Sec. II we summarize the definition of $S U(N)$ pure gauge theories in the presence of trace deformations and our lattice implementation. In Sec. II B we review the theoretical and numerical methods adopted to determine the glueball and torelon spectrum in our numerical simulations. In Sec. III we present our numerical results for $S U(3)$, which consist of determinations of glueball and torelon masses both with and without trace deformations, in order to assess a possible correspondence between the two cases, and for different values of the lattice spacing, in order to assess the relevance of cutoff effects. In Sec. IV we present some exploratory results regarding the $S U(4)$ case. Finally, in Sec. V, we draw our conclusions.

\section{NUMERICAL SETUP}

\section{A. Traced deformed Yang-Mills theory}

Trace deformed $S U(N)$ Yang-Mills (YM) theories were first proposed in Ref. [7], although a previous lattice study was already presented in Ref. [8]. Additional center symmetric couplings to the Polyakov loop are added to the usual YM action, in order to prevent center symmetry breaking, even for compactification length $l$ smaller than the critical one $l_{c}$. The action of trace deformed $S U(N) \mathrm{YM}$ theory is thus given by [7]:

$$
S^{\text {def }}=S_{\mathrm{YM}}+\sum_{\vec{n}} \sum_{j=1}^{\lfloor N / 2\rfloor} h_{j}\left|\operatorname{Tr} P^{j}(\vec{n})\right|^{2},
$$

where $\vec{n}$ identifies a point in the space orthogonal to the compactified direction, \lfloor\rfloor denotes the floor function, $P$ is the Polyakov loop in the compactified direction and the $h_{j} \mathrm{~s}$ are the deformation parameters, corresponding to the number of independent, center-symmetric functions of the Polyakov loop; 1 and 2 parameters are needed respectively for the cases $N=3$ and $N=4$ explored in the present study. $S_{\mathrm{YM}}$ is the standard YM plaquette action:

$$
S_{\mathrm{YM}}=\beta \sum_{\square}\left\{1-\frac{1}{N} \operatorname{Re} \operatorname{Tr} U_{\square}\right\} ; \quad \beta=\frac{2 N}{g^{2}} .
$$

Trace deformation and other alternatives (like the introduction of adjoint fermions or the use of nonthermal boundary conditions) have already been studied in several previous works [5,6,10-25,30-33]. The possibility of preserving center symmetry, also in the limit of vanishing compactification radius, is quite intriguing; weak coupling methods based on the small compactified direction could be used to study the confining properties of YM and the volume independence predicted by Eguchi and Kawai in Ref. [1] could be exploited. Moreover, trace deformation is also a powerful tool to investigate the connection between the realization of center symmetry and the properties typical of the low temperature, confining region. In Refs. [26,27] the $\theta$ dependence of the phase in which center symmetry is recovered has been studied both for $S U(3)$ and $S U(4)$. In both cases topological observables reach a plateau as soon as the deformation coupling $h$ is high enough to restore full center symmetry. The plateau value of such observables is also compatible with the corresponding value of the undeformed theory. This result shows, at least for topological observables, that the center stabilized phase has the same nonperturbative properties of the usual confining, zero temperature one.

\section{B. Mass extraction on the lattice}

Masses of color singlet states in lattice gauge theories can be calculated using the standard decomposition of a Euclidean correlator of some operator $\phi(t)$, with high enough overlap onto the physical states in terms of the energy eigenstates of the Hamiltonian $H$ :

$$
\begin{aligned}
\left\langle\phi^{\dagger}\left(t=a n_{t}\right) \phi(0)\right\rangle & =\left\langle\phi^{\dagger} e^{-H a n_{t}} \phi\right\rangle=\sum_{i}\left|c_{i}\right|^{2} e^{-a E_{i} n_{t}} \\
& \stackrel{t \rightarrow \infty}{=}\left|c_{0}\right|^{2} e^{-a E_{0} n_{t}},
\end{aligned}
$$

where the energy levels are ordered, $E_{i+1} \geq E_{i}$, with $E_{0}$ that of the ground state. The only states that contribute in the above summation are those that have nonzero overlaps i.e., $c_{i}=\left\langle\operatorname{vac}\left|\phi^{\dagger}\right| i\right\rangle \neq 0$. We, therefore, need to match the quantum numbers of the operator $\phi$ to those of the state we are interested in. In this work we are interested in glueballs and torelons, thus, we need to encode the right quantum properties within the operator $\phi$ which will enable us to project onto the aforementioned states.

The extraction of the ground state relies on how good the overlap is onto this state and how fast in $t$ we obtain the exponential decay according to Eq. (3). The overlap can be maximized by building operator(s) which "capture" the right properties of the state, in other words by projecting onto the right quantum numbers as well as onto the physical 
length scales of the relevant state. In order to achieve a decay behavior setting in at low values of $t$ one has to minimize contributions from excited states. To this purpose we employ the variational calculation or GEVP (generalized eigenvalue problem) [34] applied to a basis of operators built by the same lattice path albeit in several blocking levels $[35,36]$. This reduces the contamination of excitation states onto the ground state and maximizes the overlap of the operators onto the physical length scales.

\section{Extracting the scalar glueball mass}

We extract the ground state mass of the scalar glueball by making use of the variational calculation. To this purpose we employ two different operators for accomplishing the GEVP. Namely, we use the simple plaquette operator as well as the rectangular operators with size $1 a \times 2 a$ and $2 a \times 1 a$. We take linear combinations of such operators along perpendicular spatial slices so that the resulting operator has the right $0^{++}$rotational properties.

On a homogeneous cubic spatial lattice with all the spatial sizes being equal and the action being homogeneous along all spatial directions the rotational symmetry is described by the octahedral subgroup of the full rotation group. There are only five irreducible representations within this group usually labelled as $R=A_{1}, A_{2}, E, T_{1}$, $T_{2}$. These five irreducible representations have dimensions of $1,1,2,3,3$ respectively. The states one can calculate will belong to these five representations. In addition, glueball states are characterized by the discrete quantum numbers of parity $P$ and charge conjugation $C$. Hence, at finite lattice spacing the glueball states will be labeled by $R^{P C}$. As the lattice spacing tends to zero one recovers the full rotational invariance, with the states falling into the $2 J+1$ multiplets labeled by the value of angular momentum $J$. So, in principle, the glueball states can be characterized by the angular momentum $J$. The ground state characterized by $A_{1}^{++}$identifies that described by quantum numbers $J^{P C}=0^{++}$. Therefore, we will focus on the $A_{1}^{++}$representation, assuming that its ground state provides the scalar ground state glueball mass.

If we choose the length in the $x$ direction to be smaller than the other two spatial sizes and/or switch on the trace deformation, i.e., $h \neq 0$, the rotational symmetry cannot characterize the states irreducibly any more and the variational calculation built for the $A_{1}$ representation projects onto all irreducible representations of the octahedral group of rotations. Nevertheless, the ground state of the calculation still belongs to the $A_{1}^{++}$channel and, thus, reflects the scalar glueball mass. Since operators can exchange intermediate glueballs along the toroidally compactified boundaries we should also be aware of finite volume effects along the $x$ direction. Recent calculations of the glueball spectrum $[37,38]$ provide bounds below which one would expect to experience such effects.

\section{Extracting the torelon mass}

In the same manner we can extract the mass of the torelon which winds around the compactified deformed direction. We obtain the torelon mass by calculating the ground state energy $m_{T}(h, L)$ of a flux tube of length $L$ that closes on itself by winding once around the spatial compactified deformed torus. We use Eq. (3) where the operator $\phi$ is the product of $S U(N)$ link matrices taken around a noncontractible closed path that winds once around the spatial torus. The simplest such operator is the elementary Polyakov loop:

$$
\phi\left(n_{t}\right)=P\left(n_{t}\right)=\sum_{n_{y}, n_{z}} \operatorname{Tr}\left\{\prod_{n_{x}=1}^{L_{x}} U_{x}\left(n_{x}, n_{y}, n_{z}, n_{t}\right)\right\} .
$$

The above formula denotes the path ordered product of link matrices in the $x$-direction winding once around the $x$-torus. Then we sum over translations along the $x$-torus and a time slice so that we project onto zero longitudinal as well as transverse momentum respectively i.e., $\left(p_{x}, p_{y}, p_{z}\right)=(0,0,0)$.

The above operator is invariant under rotations about its torelon axis and so has angular momentum $J=0$. It is also clearly invariant under a combined parity and charge conjugation transformation $(C P)$. Therefore, this operator is ideal for projecting onto the torelon ground state. Once more we employ smearing and blocking techniques in order to enhance the projection onto the physical states. Torelon operators are not invariant under center symmetry transformations and, thus, their vacuum expectation value is zero as long as this is not broken.

\section{NUMERICAL RESULTS FOR $S U(3)$}

The main goal of this paper is to investigate how the masses of glueballs and torelons behave in the presence of a trace deformed term in the action along the $x$-circle. For this reason, in most of our simulations we consider lattices with a small fixed extent along the $x$-direction (while the rest of the lattice sizes are kept fixed to a much larger value), such that the undeformed theory stays in the deconfined phase. By switching on the trace deformations we begin to inhibit the spontaneous breaking of center symmetry: as expected, above some threshold, confinement is restored and one might expect the glueball mass to acquire a value consistent with that for $T=0$.

However, before going to the main point, we will present an extensive study of the spectrum in the nondeformed theory. The reason is that an alternative way to recover confinement, even in absence of any deformation, is by increasing the lattice size in the $x$-direction: once the length $l_{x}$ is larger than the deconfining critical length $l_{c}$, confinement is restored. Therefore, investigating how the glueball and torelon spectrum behave as a function of $l_{x}$ will set the ground for a meaningful comparison with the behavior of 
the spectrum at fixed $l_{x}$ as a function of the deformation parameter: that will permit to interpret results at nonzero $h$ in terms of an effective $l_{x}$ and will shed light on some of our findings.

\section{A. Results for the nondeformed theory}

We discuss glueball masses at first, then torelons. The latter present a nontrivial dependence on the compactification radius even in the confined phase, which is relevant to the discussion of results obtained for the deformed theory.

\section{Glueballs in the nondeformed theory}

We begin our study by performing an exploratory investigation of the glueball ground state mass. To understand how the ground state mass behaves for $h=0$ and a lattice volume with one of the lattice lengths smaller than the others, we perform a quick calculation for a sequence of increasing $L_{x}$ while keeping the other three lattice extents fixed to $L_{y}=L_{z}=L_{t}=30$. In Fig. 1 we present the ground state of $A_{1}^{++}$at $\beta=6.0$ which results from the GEVP using the simple plaquette as well as the rectangular $1 \times 2$ and $2 \times 1$ operators in 5 different blocking levels. The main features of this figure can be described in three different regimes of length $L_{x}$.

Starting from the largest value of $L_{x}$ and moving downwards, at $L_{x}=30$ the mass of our variational calculation is simply the mass of the ground state of the scalar glueball with quantum numbers $A_{1}^{++}$. The hypercubic lattice is homogeneous and therefore there are no mixings with other irreducible representations of the hypercubic group of rotations. As we start reducing the length in the $x$ direction, the variational calculation although starts projecting onto all irreducible representations of the octahedral group of rotations, its ground state is still that of the $A_{1}^{++}$

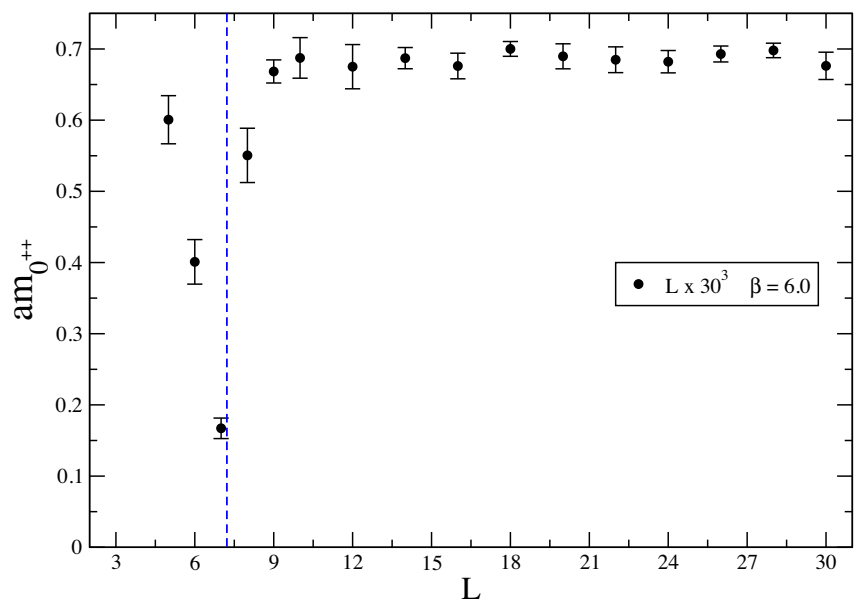

FIG. 1. The mass of the ground state of the variational calculation as a function of $L_{x}$ for $h=0$. The blue dashed vertical line corresponds to the critical value of length $L_{c}$. channel. As explained before, due to the exchange of intermediate glueballs along the toroidally compactified boundary, we should be aware of finite volume effects along the $x$-direction. Recent calculations of the glueball spectrum [37] suggest that no finite volume effects should be visible for $L_{i} \geq 20$ at $\beta=6.0$ where $i=x, y, z$. Hence, we expect that some finite volume effects might appear to show up for $L_{x}<20$. Nevertheless, within our statistical precision no such effects appear to rise and there is a well defined plateau for the glueball ground state $0^{++}$along the region $L_{x} \in[9,30]$.

As we move to smaller values of $L_{x}\left(L_{c}<L_{x}<9\right)$, due to the fact that one of the spatial directions of the lattice becomes critically small, our variational calculation can capture an interacting torelon-antitorelon (ditorelon) state which, as expected, vanishes as we approach the critical length. The mass of the state is not just equal to twice the mass of the torelon but there is a nonzero contribution due to the interaction. The appearance of such a state in the glueball calculation is not prohibited by center symmetry and therefore there is some nonzero overlap with our variational basis of operators. Of course as the number of colors $N$ increases we expect that such overlaps will be suppressed. An investigation of the glueball spectrum in the large- $N$ limit [38] will demonstrate up to what value of $N$ such states are visible. Because the main contribution in these states comes from twice the mass of the torelon for a given $L_{x}$, as expected the mass of the ground state of our variational calculation decreases with $L_{x}$. In general these states can be considered to be finite volume states which vanish as we increase all the spatial lattice sizes. In addition, given that the phase transition is weakly firstorder, close to the critical length the masses of the glueballs might be governed by the critical exponents of the theory. This means that the mass of the $0^{++}$glueball might experience a drift toward zero as we approach the critical length from above. For larger values of $N$ where the aforementioned transition is strong first order the naive expectation is that the transition does not affect the glueball mass. To provide a definite answer on what these states are a dedicated investigation should be carried out; however this goes beyond the scope of this work.

Finally as expected, when we decrease $L_{x}<L_{c}$, the calculation gives access to glueball screening masses. So, as a matter of fact one can think of the theory on a lattice of $L_{\tau} \times L^{2} \times L_{Z}$ with $L_{\tau}=L_{x}<L=L_{Z}$ and $L_{\tau}=1 / a T$ where $T$ is the temperature. Our operators are build to project onto the ground state of $0^{++}$for a calculation at $T=0$ and will, thus, capture this state. The mass appears to increase with temperature reflecting the large thermal shift of the $0^{++}$mass.

Hence, our data for $\beta=6.0$ outline three different regions in $L_{x}$, similar results have also been obtained for $\beta=6.2$. To summarize:

(1) For $l_{x}<l_{c}$ we obtain glueball screening masses. 
TABLE I. The value of the string tension calculated using the NG formula, the value of the scalar glueball mass at $T=0$ as well as the critical length for the three values of $\beta$. The critical length has been extracted by cubic spline interpolations of the results taken from Ref. [39].

\begin{tabular}{lccc}
\hline \hline$\beta$ & $a^{2} \sigma$ & $a m_{0^{++}}$ & $L_{c}$ \\
\hline 5.8 & $0.09882(79)$ & $0.8567(349)$ & $5.000(5)$ \\
6.0 & $0.04644(60)$ & $0.6763(192)$ & $7.225(19)$ \\
6.2 & $0.02487(20)$ & $0.5218(147)$ & $9.892(46)$ \\
\hline \hline
\end{tabular}

(2) For $l_{c}(\beta) \sqrt{\sigma}<l_{x} \sqrt{\sigma} \lesssim 2$ we may obtain finite volume effects such as ditorelon and glueball states influenced by virtual glueball exchanges or the glueball mass influenced by the weakly first order phase transition.

(3) For $2 \lesssim l_{x} \sqrt{\sigma}<\infty$ we extract the $0^{++}$glueball ground state.

The $0^{++}$glueball ground state, for the three values of $\beta$ considered in this work and extracted for a homogeneous spatial lattice where the octahedral subgroup of rotations is restored, are provided in Table I. These results are in good agreement with recent calculations such as Ref. [37]. A careful inspection over the gluball masses suggests that $m_{0^{++}} / \sqrt{\sigma}$ does not scale linearly with $a^{2} \sigma$, this is due to the fact that the glueball mass for the coarsest lattice acquires scaling corrections of higher power $\left(a^{4} \sigma^{2}\right)$, this can be seen clearly in Fig. 11 of Ref. [37].

\section{Torelons in the nondeformed theory}

In this case, we calculate the ground state energy $m_{T}(l)$ of a flux tube of length $l$ that closes on itself by winding once around a spatial torus of size $l$. For a theory without a center deformation and with the torelon being formed along a direction with size larger than the deconfining length $l_{c}=$ $a L_{c}$ its mass is given by the ground energy of a relativistic closed (noncritical) string. It has been demonstrated that a very good approximation for the ground state of the torelon is provided by the Nambu-Goto formula:

$$
m_{T}\left(h=0, l>l_{c}\right) \stackrel{\mathrm{NG}}{=} \sigma l\left(1-\frac{2 \pi}{3 \sigma l^{2}}\right)^{1 / 2},
$$

which derives from the light-cone quantization of the bosonic string. Physically it arises from the regularized sum of the zero-point energies of all the quantized oscillators on the string. It is known to provide an excellent approximation to the lattice calculations [40] for reasons that have now become well understood (see for instance Refs. [41,42].)

As a matter of fact one expects that this approximation works well for flux-tube lengths of $l \sqrt{\sigma}>2.5$ [40]. Thus, by fitting the ground state as a function of the length $l$ to this expression we can extract the string tension $\sigma$. The value of the string tension for each value of $\beta$ is listed in Table I.

The spectrum of the ground state as well as the low-lying spectrum of the torelon has been investigated extensively [40] and our results are in good agreement with previous findings. Hence, this part of our study does not provide new knowledge. Nevertheless, it provides the energy scale for each different value of $\beta$ we consider in our work as well as a comparison with the spectrum of the torelons for the trace deformed case; we will comment on that in Sec. III B 3. In Fig. 2 we present the ground state mass of the torelon $m_{T} / \sqrt{\sigma}$ as a function of its length $l \sqrt{\sigma}$ for the three values of $\beta$.

\section{B. Results for the trace deformed theory}

We turn now to the case of the trace deformed theory. Practically, we kept $L_{x}<L_{c}$ as well as $L_{y}, L_{z}, L_{t} \gg L_{c}$, and we changed $h$. For most simulations we fixed $a L_{x} \simeq$ $0.54 \mathrm{fm}$ exploring different lattice spacings, considering in particular $L_{x}=4,6,8$ for $\beta=5.8,6.0,6.2$ respectively, with $L_{y}=L_{z}=L_{t}=30$ in all cases. In addition, we performed simulations for $L_{x}=6$ at $\beta=6.2$ and $L_{x}=8$ at $\beta=6.1$ in order to explore different values of the compactification length, respectively $l_{x} \simeq 0.4 \mathrm{fm}$ and $l_{x} \simeq 0.62 \mathrm{fm}$.

Measurements of glueball and torelon states were taken along each of the homogeneous directions, in order to increase statistics, using around 4000 decorrelated configurations for each value of $h$.

\section{Glueballs in the trace deformed theory}

Similarly to the case of the nondeformed theory, we performed a measurement of the glueball scalar mass using the variational technique with a basis of operators built out of the simple plaquette as well as the rectangular operators,

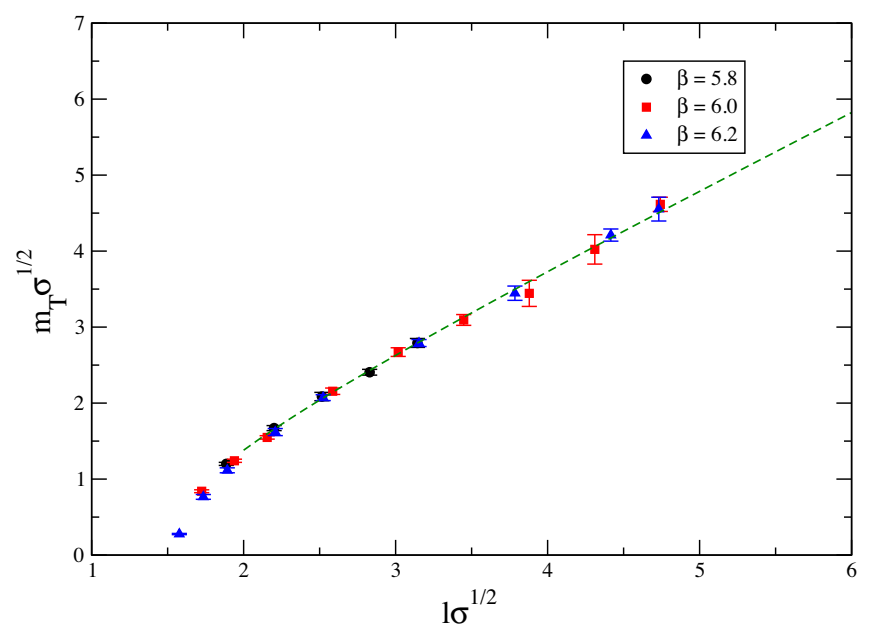

FIG. 2. The mass of the torelon in units of $\sqrt{\sigma}$ as a function of the flux-tube length in dimensionless units. The line corresponds to the Nambu-Goto formula given in Eq. (5). 
using smearing and blocking techniques in order to enhance the overlap onto the physical states. We extracted the absolute ground state of the glueball mass which corresponds to the $0^{++}$ground state as a function of $h$ for three values of the lattice spacing, i.e., three values of $\beta$. Results obtained for $l_{x} \simeq 0.54 \mathrm{fm}$ are shown in Fig. 3 where
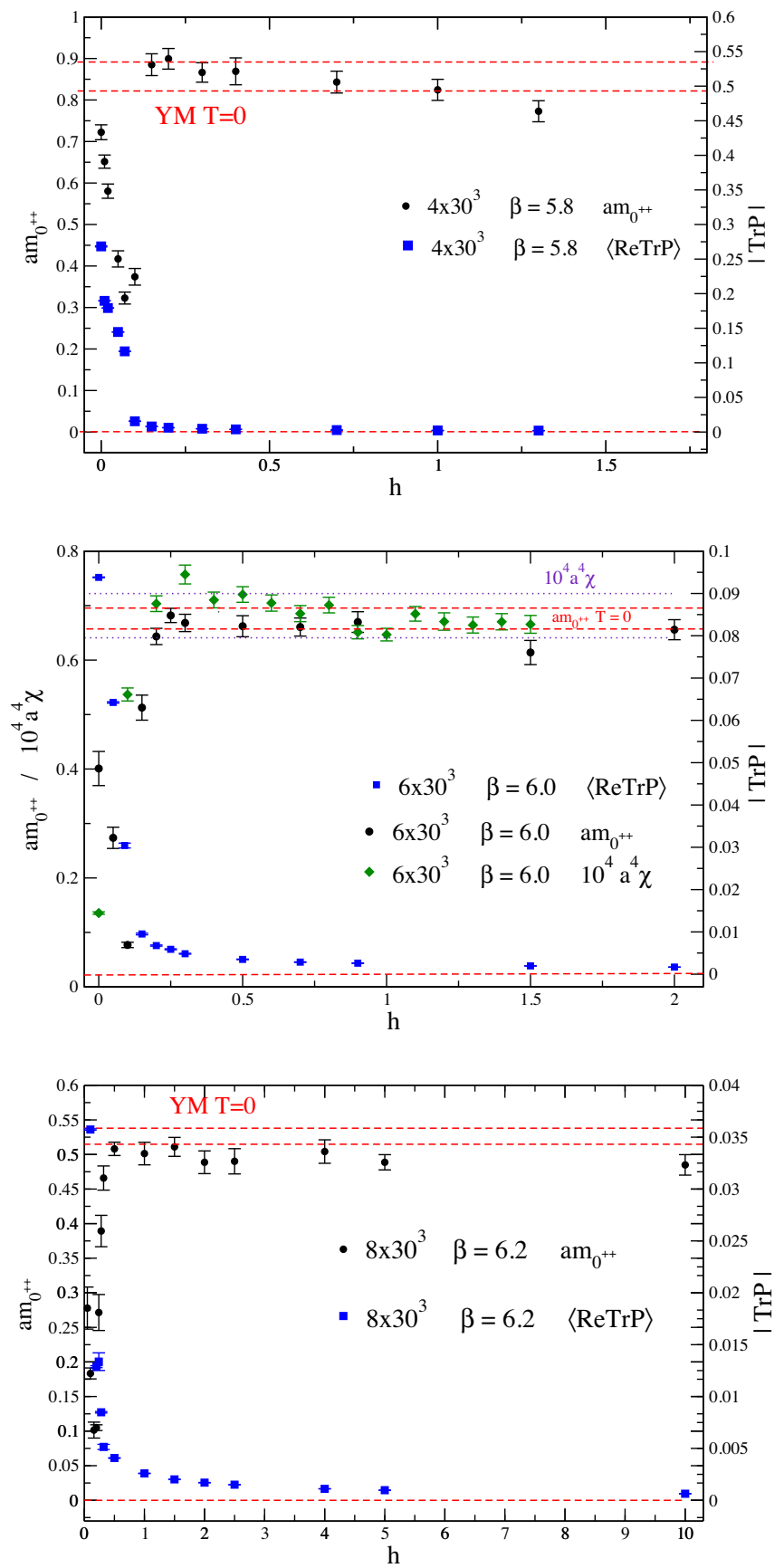

FIG. 3. The mass of the ground state resulting out of the variational calculation for glueballs (in black) as well as the average value of the Polyakov loop (in blue) in the trace deformed theory as a function of $h$ and the three values of $\beta$. For $\beta=6.0$ we also report, for comparison, results for the topological susceptibility, which have been taken from Ref. [26]. we also report, for comparison, the expectation value of the Polyakov loop, which becomes zero once the center symmetry is restored.

The plots resemble to an adequate extent the behavior of the glueball mass observed for the case of the nondeformed theory, where we alter the lattice size along the $x$ direction. For very small values of $h$ center symmetry is still broken, so that the variational calculation captures the screening mass. As we keep increasing $h$, center symmetry gets restored and the theory experiences a transition to confinement, so that the correlators start capturing confining states such as bound states of two closed flux-tubes or glueball states: the resulting glueball mass shows a dip after which, deep in the confined phase, it reaches a well defined plateau. This plateau appears to be consistent with the glueball mass extracted at $T=0$. The critical value of $h$, as expected, depends on $\beta$, i.e., on the lattice spacing. Analogous results have been obtained for the other explored values of $l_{x}$ : the plateau values in the reconfined phase are summarized in Table II.

It is interesting to notice that this behavior resembles closely that of the topological susceptibility $\chi$, which is reported for comparison in one case $(\beta=6.0)$ and reaches a plateau at approximately the same values of $h$ : a similar behavior is observed for the other cases. That shows that the reconfined compactified theory recovers most of the nonperturbative features of the original $T=0$ theory at the same time.

Just for $\beta=6.2$ we report results extended to larger values of the deformation parameter, namely up to $h=10$, showing that the glueball mass in this case is also quite stable: this is an interesting aspect, however it is not essential and indeed the plateau is not so stable for other values of the lattice spacing. In general, we are more interested in the small $h$ region, where the properties of the reconfined phase can be compared to those of the standard confined phase more closely, trying also to determine a sort of $h$-dependent effective compactification radius, as we illustrate in more details in the next paragraph.

\section{Trying to match the h-dependence of the deformed theory to the l-dependence of the undeformed theory}

The close resemblance between the dependence of the glueball mass on $l$ at $h=0$, and of that on $h$ at fixed $l$,

TABLE II. The plateau value of $a m_{0^{++}}$for the different lattice setups used.

\begin{tabular}{lccc}
\hline \hline$\beta$ & $L_{x}$ & $l_{x}[\mathrm{fm}]$ & $a m_{0^{++}}$ \\
\hline 5.8 & 4 & 0.54 & $0.85(4)$ \\
6.0 & 6 & 0.56 & $0.66(2)$ \\
6.2 & 6 & 0.40 & $0.46(2)$ \\
6.1 & 8 & 0.62 & $0.50(2)$ \\
6.2 & 8 & 0.54 & $0.59(2)$ \\
\hline \hline
\end{tabular}


suggests a possible interpretation of a nonzero $h$ in terms of an effective, $h$-dependent compactification size $l_{\text {eff }}(l, h)$, i.e., a match of results at fixed $l$ and $h \neq 0$ to those obtained with a compactification size $l_{\text {eff }}$ at $h=0$. In order to further test this possibility, we have worked on a possible ansatz for such dependence.

Since a positive/negative $h$ will favor/disfavor confinement, this is analogous to increasing or decreasing $l$. Hence, the dependence of such $l_{\text {eff }}$ on $h$ must be odd at least at the lowest order in a Taylor expansion around $h=0$. On the other hand, it is sensible to look for a description in terms of $l_{\text {eff }}$ only for small values of $h$, since large values are expected to modify the theory more substantially.

Therefore, we have considered the following linear ansatz

$$
l_{\mathrm{eff}}(l, h)=l(1+A h)
$$

where $A$ is a dimensionless parameter, which in general is expected to depend on the ultraviolet (UV) cut-off since $h$ is a bare parameter.

Such ansatz turns out to work reasonably well. In Fig. 4 we compare results obtained for $\beta=6.0$, either at $h=0$ and variable $L_{x}$ or at fixed $L_{x}=6$ and variable $h$, showing that a reasonable match is obtained by taking $A \simeq 2.10$, with an uncertainty estimated around $10 \%$ based on a by eye matching. In Fig. 4 we report additionally also results obtained for $\beta=6.2$ and $L_{x}=8$, just to show that in this case $A \simeq 1.35$, confirming that it is indeed cutoff dependent.

From Fig. 4 one could get the wrong suggestion that the ansatz works well even for large values of $h$. However one should consider that, once in the confined phase, the glueball mass is in fact $l$-independent (hence volumeindependent), even in the undeformed theory. A better

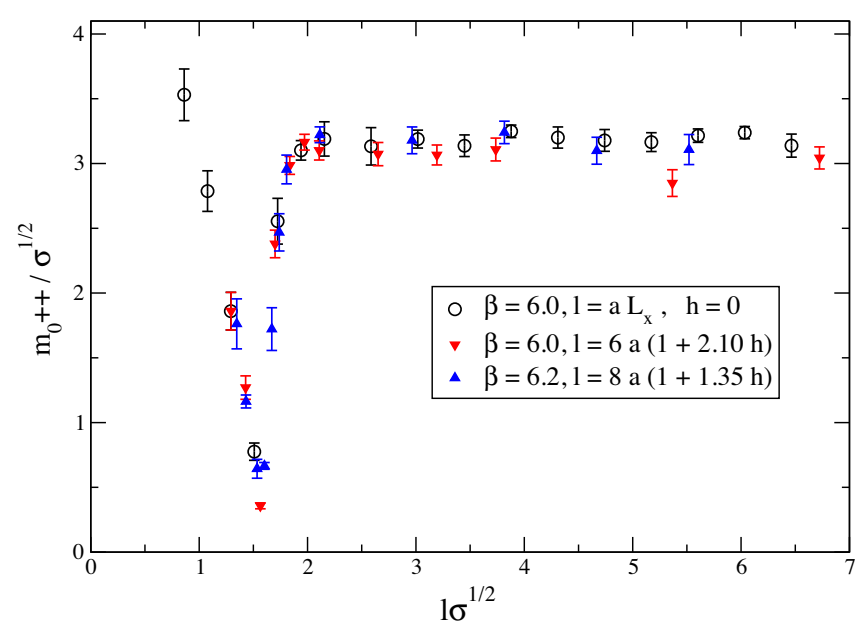

FIG. 4. Gueball masses obtained either at $h=0$ and variable $l$ or at fixed $L_{x}$ and variable $h$. In the latter case the horizontal scale has been fixed according to the ansatz in Eq. (6), where the constant $A$ has been tuned based on a by eye matching. feeling about the range of validity of the ansatz in Eq. (6) is therefore obtained by looking at the torelon mass, which turns out to have a nontrivial dependence on $l$ in the confined phase.

\section{Torelons in the trace deformed theory}

In order to discuss the behavior of the torelon ground energy as a function of the deformation parameter, we start by illustrating the results obtained at $\beta=6.0$ and $L_{x}=6$, which are reported in Fig. 5 and compared with results obtained in the undeformed theory, adopting the same matching in terms of an effective compactification size fixed from the glueball mass.

It is clear that the matching works pretty well also for torelon masses as long as $h$ is small (in particular for $h \lesssim 0.2)$. Then deviations are larger and larger and, contrary to the linearly rising behavior observed in the undeformed theory, results seem to reach a well defined plateau at asymptotically large values of $h$. A similar behavior is observed also in other cases, as an example in Fig. 6 we show the ground mass of the torelon as a function of $h$ for different values of the lattice spacing and $l \simeq 0.54 \mathrm{fm}$. Assuming that the ground energy indeed reaches a plateau with $h$, we have tried to fit data in Fig. 6 according to the following ansatz

$$
a m_{T}(h)=a m_{T}(\infty)+b e^{-c h} .
$$

The fit works well, i.e., with a $\chi^{2} /$ d.o.f. of order $\sim 1$, if data at the lowest values of $h$ are discarded.

It is interesting to ask what the plateau values should be compared with. Since large values of $h$ tend to suppress local fluctuations of the Polyakov loop more and more, it is reasonable to look for predictions obtained in the

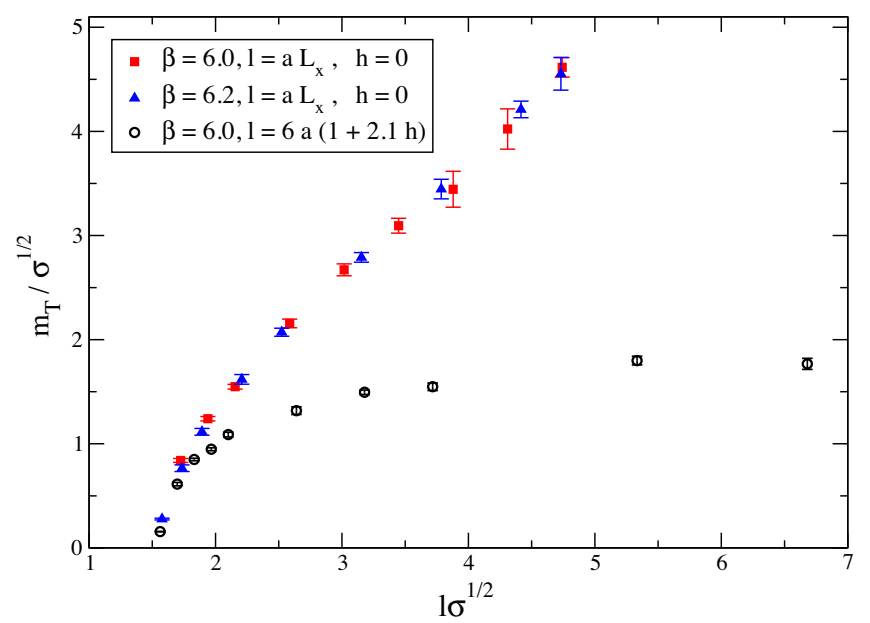

FIG. 5. A comparison similar to that of Fig. 5, performed in this case for the torelon masses. The rescaling parameter $A$ has been kept fixed to the value already matched for the glueball masses, in order to see if the ansatz in Eq. (6) works well for all observables, at least for small values of $h$. 
semiclassical regime, such as those reported in Ref. [7]. When we switch on the trace deformation along a circle of small radius, that is to say $l_{x} \ll 1 / \sqrt{\sigma}$, the trace potential in the action becomes minimum if the Polyakov loop along this circle acquires a diagonal value of $P=\operatorname{Diag}\left(1, e^{2 \pi i / N}, e^{4 \pi i / N}, \ldots, e^{4 \pi i(N-1) / N}\right)$ up to conjugation by an arbitrary $S U(N)$ matrix. If one works in a gauge in which the Polyakov loop $P$ is diagonal, and using gauge-dependent language, this configuration may be regarded as breaking the gauge symmetry down to the maximal Abelian subgroup i.e., $S U(N) \rightarrow U(1)^{(N-1)}$.

The modes of the diagonal components of the $S U(N)$ gauge field with no relative momentum along the compactified direction describe photons associated with the $S U(N)$ Cartan subgroup while modes with nonzero relative momentum form a Kaluza-Klein tower with masses being integer multiples of $2 \pi / L$ [7]:

$$
m_{T}(l)=\frac{2 \pi}{N l}=\frac{2 \pi}{3 l} .
$$

Such prediction should work in principle for $N \Lambda l \ll 1$, where $\Lambda$ is the QCD scale, which is not the case for our simulations. However, as a matter of fact, the results of Fig. 6 show that the plateau values are quite close to the $2 \pi / 3 l$ prediction, even if $l$ is only slightly smaller than the deconfining length $l_{c}$. One should consider that, in this case, we are discussing a sort of large-deformation limit of what is found, and this could have some influence on the final findings.

In Fig. 7 we report the three plateau values of Fig. 6 for $a m_{T} L$ as a function of $1 / L^{2}$. Mind that $1 / L^{2}=a^{2} / l^{2}$ with $l$ being fixed in physical units, thus this plot corresponds to an extrapolation of $a m_{T} L$ to the continuum limit and shows two interesting things at the same time. First, the three points can be adequately fitted with a straight line, i.e., assuming that UV corrections are $O\left(a^{2}\right)$, so that the plateau value corresponds to a well-defined continuum quantity which we derive to be $a m_{T} L=2.1(2)$. Second, this number is in good agreement with the theoretical expectation in the semiclassical regime, i.e., $2 \pi / 3 \simeq 2.094$.

However, in order to test if this is just a fortuitous coincidence, we have decided to repeat our study for two different values of $l$, one smaller and one larger. In particular, we explored the case $L_{x}=6$ at $\beta=6.2$, corresponding to $l \sim 0.4 \mathrm{fm}$, and the case $L_{x}=8$ at $\beta=6.1$, corresponding to $l \sim 0.62 \mathrm{fm}$. It is not easy, in particular, to go to smaller values, since it is difficult to keep lattice artifacts well under control as $l$ is reduced with a fixed amount of lattice spacings $L_{x}$ in the compactified direction (see Ref. [43] for a discussion on this point).

In Fig. 8 we report, as a function of $h$, values obtained for $l \sim 0.4 \mathrm{fm}$ for both the glueball and the torelon mass. One can see that the plateau region for the glueball mass is somewhat reduced in this case (we consider the region up to $h \simeq 1$ ), while the large- $h$ plateau for the torelon mass is still
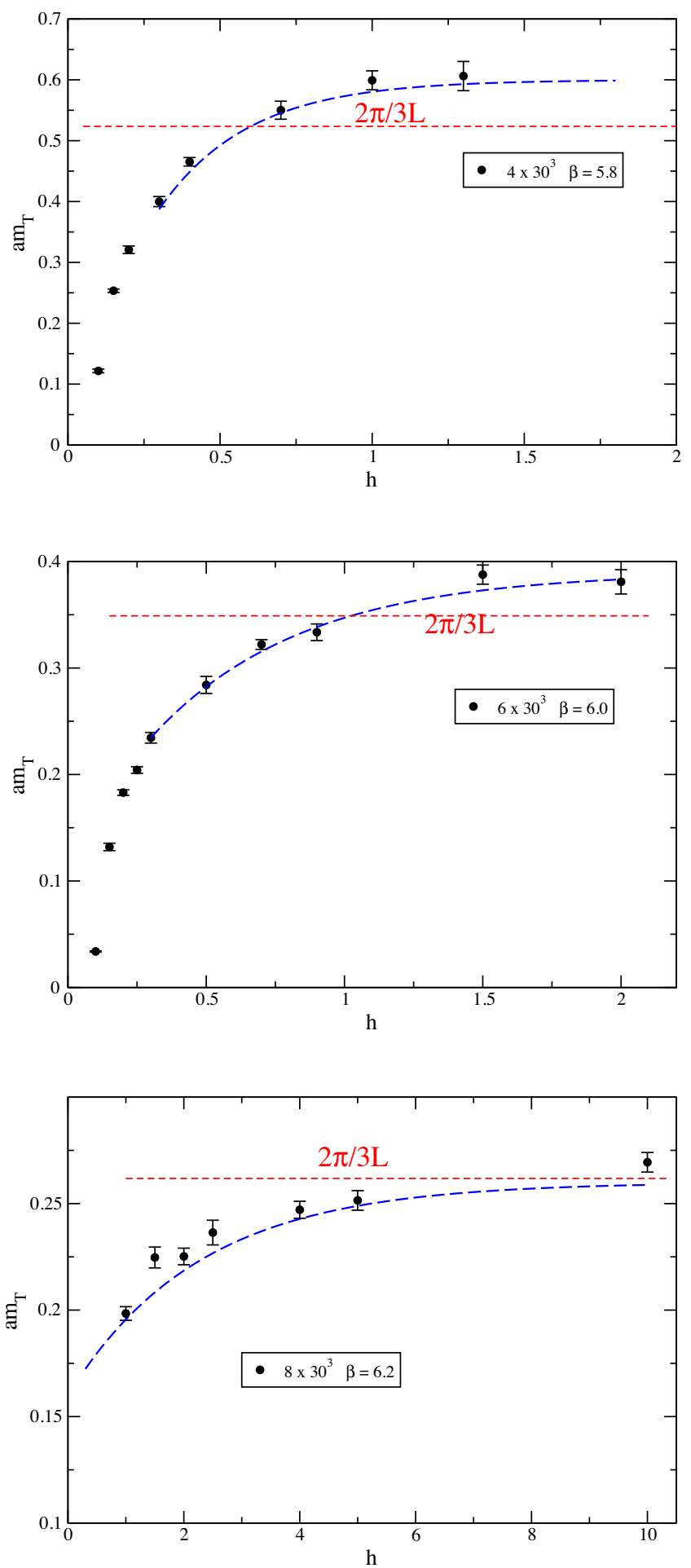

FIG. 6. The mass of the ground state resulting out of the variational calculation for torelons in the trace deformed theory as a function of $h$ and the three values of $\beta$.

well defined. The plateau values for these new values of $l$ are reported in Fig. 9, where they are compared with those previously obtained for $l \simeq 0.54 \mathrm{fm}$, with results obtained for the glueball masses and with the exploratory results obtained for $S U(4)$ which are discussed in the next section. 


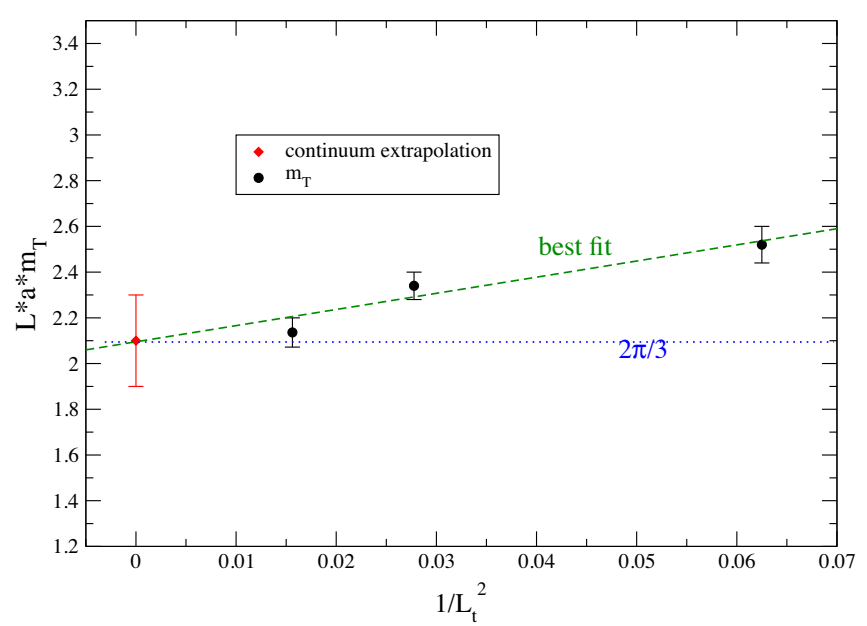

FIG. 7. The continuum extrapolation of the torelon masses extracted by fitting the ansatz of Eq. (7) on the torelon data for the three values of $\beta$. The dotted line represents the theoretical prediction $m_{T}=2 \pi / 3$ [7], while the dashed line represents the results of a continuum extrapolation assuming $O\left(a^{2}\right)$ corrections, which yields a $\chi^{2} /$ d.o.f. $=1.7 / 1$.

One can appreciate that the glueball mass does not show, within errors, a significant dependence on $l$, while the torelon mass has a well defined dependence which is still compatible with the $2 \pi /(3 l)$ prediction, apart from the point at the largest explored value of $l$. Of course it would be interesting, in future studies, to extend this investigation to smaller values of $l$, possibly to the point where the torelon mass and the glueball mass cross each other.

\section{SU(4)}

The extension of our study to $S U(4)$ in this context is exploratory and aimed at enlightening the main differences

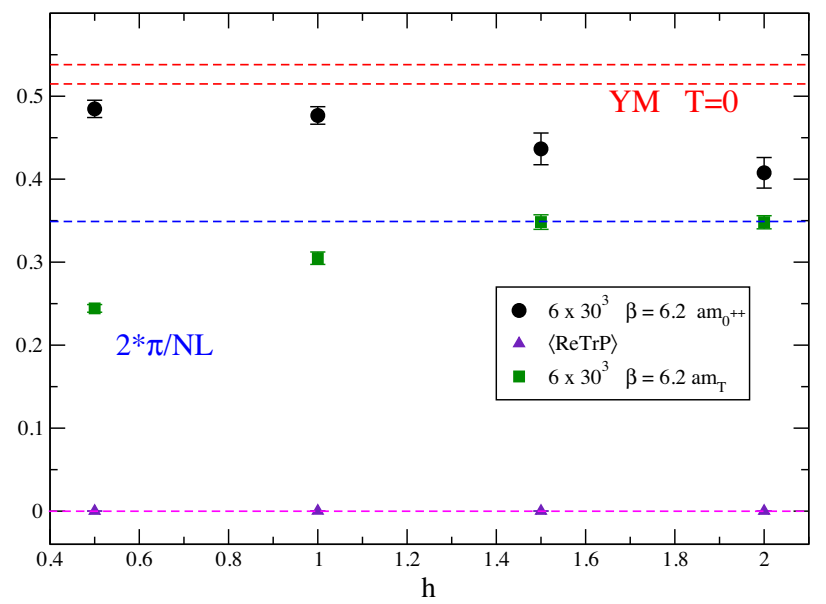

FIG. 8. Glueball and torelon masses, as a function of $h$, for $L_{x}=6$ and $\beta=6.2$, corresponding to $l \simeq 0.4 \mathrm{fm}$. We report also values obtained for the Polyakov loop expectation value, showing that center symmetry is restored for all explored values of $h$.

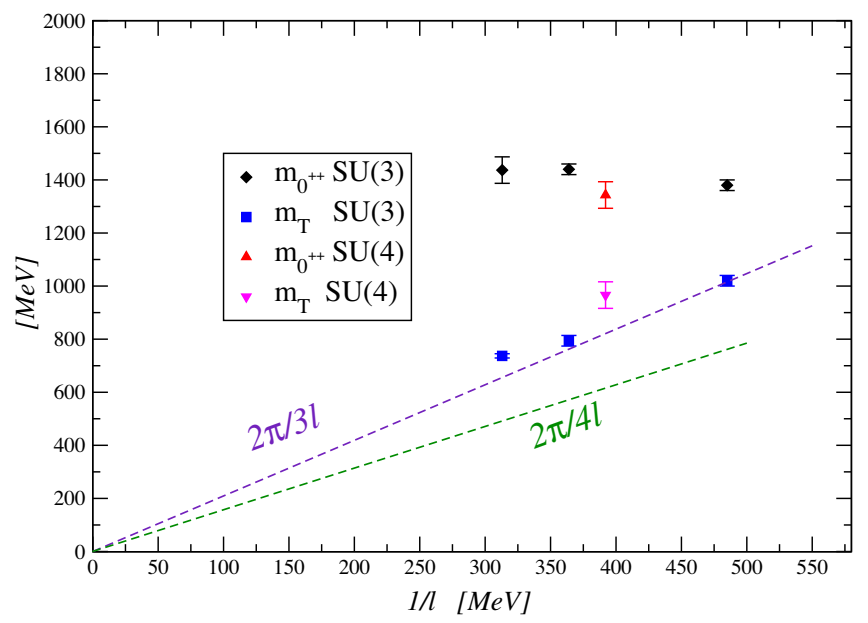

FIG. 9. Glueball and torelon masses as a function of $1 / l$ for both $S U(3)$ and $S U(4)$. The dashed lines represent the prediction from Ref. [7].

emerging when moving to $N>3$; in particular, we expect to go even farther from the semiclassical regime dictated by $N \Lambda l \ll 1$, so that, in principle, the matching of torelon masses to the prediction of Eq. (8) should be worse.

As explained in Sec. II, in this case one can introduce two different deformation parameters, $h_{1}$ and $h_{2}$, leading to a more structured phase diagram, which has been sketched in Ref. [27] and is characterized by different phases where center symmetry is completely broken, partially broken, or completely restored. In this study we consider only the case of the diagonal deformation $h=h_{1}=h_{2}$, which guarantees a complete restoration of center symmetry for large enough $h$ and is thus adequate to our purposes.

We have also considered a single value of the compactification size, $l \simeq 0.50 \mathrm{fm}$ corresponding to a deconfined phase in the undeformed theory, at a fixed value of the UV cutoff, $a \simeq 0.083 \mathrm{fm}$, with no aim toward an extensive

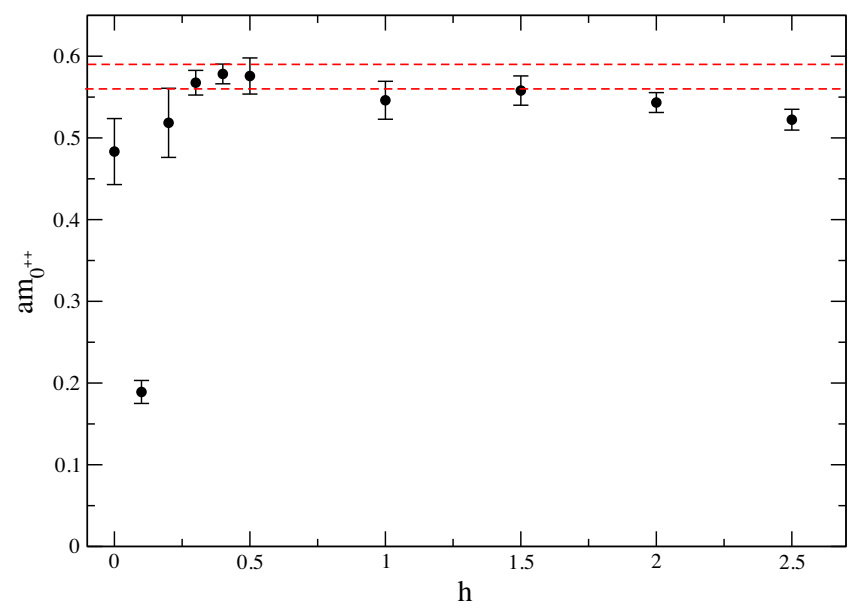

FIG. 10. Glueball mass for $\beta=11.15$ on a $6 \times 32^{3}$ lattice with both the deformations switched on, $h_{1}=h_{2}=h$. 


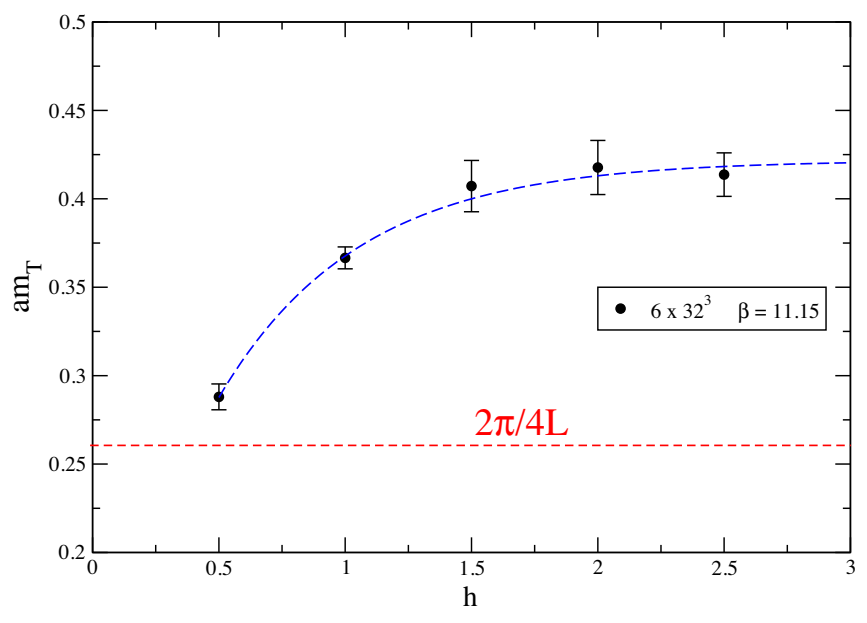

FIG. 11. Torelon mass for $\beta=11.15$ on a $6 \times 32^{3}$ lattice as a function of $h=h_{1}=h_{2}$.

study of $l$-dependence or continuum extrapolation. Results for the glueball mass as a function of $h$ are presented in Fig. 10: the behavior resembles closely that already observed for $S U(3)$, reaching a well defined plateau for $h \gtrsim 0.5$, which corresponds to a phase where center symmetry is completely restored [27]. Moreover, the plateau value is in good agreement with the $T=0$ value, which is reported in the figure as well. The $T=0$ result has been computed on an isotropic lattice with volume of $30^{4}$, with the same value of $\beta$ and it is consistent with results from Ref. [38].

Also results for the torelon ground state, which are presented in Fig. 11, show a behavior quite similar to that observed in $S U(3)$, with an approach to a plateau value at large values of $h$ which also in this case has been fitted using the ansatz reported in Eq. (7). However, in this case the plateau value turns out to be significantly larger than the semiclassical prediction of Eq. (8): this is more clear from Fig. 9, where the results obtained in $S U(4)$ for both the glueball mass and the torelon mass are compared with the $S U(3)$ results.

\section{DISCUSSION AND CONCLUSIONS}

The purpose of this investigation was that of exploring the properties of trace deformed Yang-Mills theories from the point of view of their physical spectrum. Such theories are defined on a space-time with at least one small compactified spatial direction and in the presence of trace deformations which prevent the breaking of center symmetry, giving the possibility to check the conjecture of volume independence for $S U(N)$ gauge theories in the large- $N$ limit. Lattice simulations have already provided successful confirmations at moderate values of $N=3,4$ for what concerns the topological properties of the theory [26,27].

Extending the investigation to the physical states of the theory permits to better clarify the relation with the standard large-volume theory, by distinguishing states in the deformed theory which have a clear correspondence with the standard physical states of the original theory, and states which are linked to the large energy scale associated with the small compactified direction and are expected to decouple as the compactification radius goes to zero. Having this in mind, we have performed a numerical study of trace deformed $S U(3)$ and $S U(4)$ gauge theories, focusing on two kinds of physical states: scalar glueballs and torelons defined around the compactified direction. The investigation has been performed for different values of the compactification size and, just for one case, using also different values of the lattice spacing, in order to check the absence of significant cutoff effects and perform a continuum extrapolation.

The study of scalar glueballs has fully confirmed what already observed for the case of $\theta$-dependence, i.e., a striking quantitative agreement with the values of the original large volume theory, which is observed as soon as the deformation is strong enough to make center symmetry unbroken. This is a further confirmation of volume independence, already observed even for moderate values of $N$ in the case of topological observables.

Further insight has been achieved by a comparison of results for the glueball mass obtained at fixed compactification size $l$ as a function of the deformation parameter $h$, with results obtained in the undeformed theory as a function of $l$. In particular, we have shown that results in the deformed theory can be interpreted in terms of an $h$-dependent effective compactification size, $l_{\text {eff }}(l, h)$, see in particular Eq. (6) and Fig. 4.

Torelons have shown a different behavior. The matching with results from the undeformed theory in terms of an effective compactification size works well only for small values of $h$, see Fig. 5. The different behavior, with respect to glueballs, can be easily understood in terms of the fact that the torelon mass is not volume independent even in the confined phase, where it increases linearly with $l$, see Fig. 7.

The last consideration sheds also light on striking realization of volume independence that is observed, both for glueballs and for topological observables, even for moderate values of $N$. Such quantities are already practically volume independent in the underformed theory, as long as center symmetry is safe, in the sense that they are in fact temperature independent till the deconfinement temperature is approached from below.

On the other hand, we have shown that the torelon mass approaches a well-defined plateau value in the large $h$ limit, which moreover has a well defined continuum limit. We have compared such value with the available semiclassical prediction [6,7] for the lowest mass of the tower of KaluzaKlein modes associated with the compactified direction, which is $2 \pi /(N l)$, hence $2 \pi / 3 l$ for $S U(3)$, where $l$ is the length of the compactified direction in physical units. 
While the $1 / l$ factor is a generic expectation for KaluzaKlein modes, the additional $1 / N$ factor is directly related to the way in which center symmetry is expected to be restored, in particular to the fact that the holonomy eigenvalues are evenly distributed around the complex unit circle and to the $S U(N) \rightarrow U(1)^{N}$ Higgsing of the theory. Such prediction is expected to work only when $N \Lambda l \ll 1$, where $\Lambda$ is the strong interaction scale, meaning $l \ll 1 / N$ in fermi units. Nevertheless, we have observed a reasonable agreement with $S U(3)$ results even for $l$ as large as $0.4-0.5 \mathrm{fm}$, while some deviations are visible for $l \gtrsim 0.6 \mathrm{fm}$; significant deviations instead emerge going to larger values of $N$, in particular for the $N=4$ and $l \sim 0.5 \mathrm{fm}$ case explored in this study.

To summarize, our numerical study provides, on one hand, a solid support to predictions regarding the way in which center symmetry is restored in the trace deformed theory. On the other hand, it fully confirms that, independently of the particular way the restoration is achieved, the small volume unbroken theory is indistinguishable from the original large volume theory for a large set of physical observables, going from $\theta$-dependence to the spectrum of glueball states. Future studies could further extend such evidence by considering smaller values of $l$, larger values of $N$ and different relevant observables.

\section{ACKNOWLEDGMENTS}

We would like to thank Mithat Unsal, Aleksey Cherman and Mike Teper for fruitful discussions. A. A. has been financially supported by the European Union's Horizon 2020 research and innovation programme "Tips in SCQFT" under the Marie Skłodowska-Curie Grant Agreement No. 791122. Numerical simulations have been performed at the Scientific Computing Center at INFN-PISA and on the MARCONI machine at Consorzio Interuniversitario del Nord-Est per il Calcolo Automatico (CINECA), based on the agreement between INFN and CINECA (under Projects No. INF19_npqcd and No. INF20_npqcd).
[1] T. Eguchi and H. Kawai, Phys. Rev. Lett. 48, 1063 (1982).

[2] L. G. Yaffe, Rev. Mod. Phys. 54, 407 (1982).

[3] G. Bhanot, U. M. Heller, and H. Neuberger, Phys. Lett. 113B, 47 (1982).

[4] A. Gonzalez-Arroyo and M. Okawa, Phys. Lett. 120B, 174 (1983); Phys. Rev. D 27, 2397 (1983).

[5] P. Kovtun, M. Unsal, and L. G. Yaffe, J. High Energy Phys. 06 (2007) 019.

[6] M. Unsal and L. G. Yaffe, J. High Energy Phys. 08 (2010) 030 .

[7] M. Unsal and L. G. Yaffe, Phys. Rev. D 78, 065035 (2008).

[8] J. C. Myers and M. C. Ogilvie, Phys. Rev. D 77, 125030 (2008).

[9] D. J. Gross, R. D. Pisarski, and L. G. Yaffe, Rev. Mod. Phys. 53, 43 (1981).

[10] M. Unsal, Phys. Rev. Lett. 100, 032005 (2008).

[11] M. Unsal, Phys. Rev. D 80, 065001 (2009).

[12] M. Shifman and M. Unsal, Phys. Rev. D 78, 065004 (2008).

[13] J. C. Myers and M. C. Ogilvie, J. High Energy Phys. 07 (2009) 095.

[14] G. Cossu and M. D’Elia, J. High Energy Phys. 07 (2009) 048.

[15] P. N. Meisinger and M. C. Ogilvie, Phys. Rev. D 81, 025012 (2010).

[16] E. Thomas and A. R. Zhitnitsky, Phys. Rev. D 86, 065029 (2012).

[17] E. Poppitz, T. Schaefer, and M. Unsal, J. High Energy Phys. 10 (2012) 115.

[18] E. Thomas and A. R. Zhitnitsky, Phys. Rev. D 87, 085027 (2013).
[19] E. Poppitz, T. Schaefer, and M. Unsal, J. High Energy Phys. 03 (2013) 087.

[20] T. Misumi and T. Kanazawa, J. High Energy Phys. 06 (2014) 181.

[21] M. M. Anber, E. Poppitz, and B. Teeple, J. High Energy Phys. 09 (2014) 040.

[22] A. Bhoonah, E. Thomas, and A. R. Zhitnitsky, Nucl. Phys. B890, 30 (2015).

[23] A. Cherman, S. Sen, M. L. Wagman, and L. G. Yaffe, Phys. Rev. D 95, 074512 (2017).

[24] T. Sulejmanpasic, Phys. Rev. Lett. 118, 011601 (2017).

[25] M. M. Anber and A. R. Zhitnitsky, Phys. Rev. D 96, 074022 (2017).

[26] C. Bonati, M. Cardinali, and M. D’Elia, Phys. Rev. D 98, 054508 (2018).

[27] C. Bonati, M. Cardinali, M. D’Elia, and F. Mazziotti, Phys. Rev. D 101, 034508 (2020).

[28] R. Falcone, R. Fiore, M. Gravina, and A. Papa, Nucl. Phys. B785, 19 (2007).

[29] B. Grossman, S. Gupta, U. M. Heller, and F. Karsch, Nucl. Phys. B417, 289 (1994).

[30] K. Aitken, A. Cherman, E. Poppitz, and L. G. Yaffe, Phys. Rev. D 96, 096022 (2017).

[31] Y. Tanizaki and M. Unsal, J. High Energy Phys. 03 (2020) 123.

[32] E. Itou, J. High Energy Phys. 05 (2019) 093.

[33] G. Bergner, S. Piemonte, and M. Unsal, J. High Energy Phys. 11 (2018) 092.

[34] M. Luscher and P. Weisz, Nucl. Phys. B240, 349 (1984).

[35] M. Teper, Phys. Lett. 183B, 345 (1987); Phys. Lett. 185B, 121 (1987). 
[36] B. Lucini, M. Teper, and U. Wenger, J. High Energy Phys. 06 (2004) 012.

[37] A. Athenodorou and M. Teper, J. High Energy Phys. 11 (2020) 172.

[38] A. Athenodorou and M. Teper, arXiv:2106.00364.

[39] B. Lucini, M. Teper, and U. Wenger, J. High Energy Phys. 01 (2004) 061.
[40] A. Athenodorou, B. Bringoltz, and M. Teper, J. High Energy Phys. 02 (2011) 030.

[41] O. Aharony and Z. Komargodski, J. High Energy Phys. 05 (2013) 118.

[42] S. Dubovsky, R. Flauger, and V. Gorbenko, J. Exp. Theor. Phys. 120, 399 (2015).

[43] C. Bonati, M. Cardinali, M. D'Elia, and F. Mazziotti, Proc. Sci., LATTICE2019 (2019) 084. 